\title{
Editorial
}

\section{Should we Use JCR Impact Factor or We Can Have Our Own Relative Importance Metrics?}

\author{
Imtiaz Arif*
}

Higher Education Commission (HEC) of Pakistan has been endeavoring to raise the quality of research in Pakistan and they have selected research journals as a tool to enhance research capabilities. Every year a team of HEC's eminent scholars evaluate and approve list of research journals which meet the HEC criteria for recognition. One criterion for the evaluation of journal is bibliometric indicators. Though different bibliometric indicators are available but HEC uses Institute for Scientific Information (ISI)Impact Factor, to evaluate Higher Education Institute (HEI), scientific research, entire journals, and individual articles.

Understanding the ISI Impact Factor and the reason why having one is so important to an academic journal such as Journal of Management Sciences is important. ISI was founded in the 1960s, acquired by Thomson in 1992 and is now is part of the Thomson Reuters Corporation, a multinational media and information firm.

The ISI maintains a database of the publishing and citation activity of over 14,000 academic journals. Each year, it publishes Journal Citation Reports which, among other metrics, list an Impact Factor for each journal in the database. The Impact Factor is a relatively straightforward metric based on a rolling average of the total number of recorded citations, divided by the total number of papers published over the most recent two-year period. In other words if all the papers published in a journal get one citation in each of the past two years, the journal would have an Impact Factor of 1.00.

Although other indexing services have created different methods to rate journal activity and value, it is the ISI Impact Factor that has become the worldwide de facto principal indicator of perceived journal quality. Consequently, academics will be under pressure to publish in journals with ISI Impact Factors and, where possible, in journals that have high ISI Impact Factors. This is based on the premise that there is a correlation between perceived journal quality and the quality of the papers within. In many cases, there will be little or no academic credit given for publishing in a journal that does not have an ISI Impact Factor.

Currently the Journal of Management Sciences (JMS) was added to ISI Master Journal List and it can be tracked on the ISI database in Emerging Source Citation Index (ESCI). Getting a journal into ISI database was not an easy task. Thomson Reuters employ strict criteria on which journals are admitted; their acceptance rate is currently at about $10 \%$ of all journals that apply. Now getting a journal into Journal Citation Report (JCR) database

\footnotetext{
*Assistant Professor, Department of Management Scienses, IQRA University. E-mail: arif.i@iuk.edu.pk
} 
with a good impact factor is our next challenge which is again not a simple task.

One of the steps to get into JCR is to produce and adhere to a consistent publication schedule which is March and October each year in case of JMS. Other main steps needed relate to the number of papers being published and the number of citations those papers are generating in other publications which are listed in ISI Mater Journal List. Both of these aspects are, of course, tied into the value of the journal as a platform to publish through and this is where we generate a nice circular argument. To be admitted into ISI-JCR database, the journal definitely needs to carry more papers and of a quality that will, in turn, generate a higher citation rate. However, given the increasing pressures on academics to publish selectively, the journal is always going to struggle to attract such submissions while it lacks an ISI Impact Factor. Persuading academics to publish citable papers in a journal that does not have ISI rating will continue to be a challenge.

Moreover, ISI databases barely cover National journals of countries, although in locallyoriented areas they play an important role in the dissemination of research, such as Social Sciences, Earth Sciences or Clinical Medicine. In the analysis of research journals in these areas, it is considered important to complement data from ISI databases with data from national sources. Therefore we need to develop our own databases that would cover Pakistani journals recognized by HEC in all disciplines. An impediment to this measure is the factor that there is no qualitative indicator like the JCR impact factor to assess prestige or quality of journals in Pakistan. It is recommended to construct impact factor measures for these national journals not covered by JCR. While it could be a laborious and time consuming task, but a citation database of Pakistani journals is inevitable. Consequently efforts should be made in creating our own journals' quality metrics and the impact factor of these journals should be calculated. It is recommended to construct impact factor measures for these national journals not covered by JCR. While it could be a laborious and time consuming task, but a citation database of Pakistani journals is inevitable.

To develop its own database Geist Science Publication (IQRA University) has developed Geist Science Relative Importance (GSRI) measures of an academic journal. This measure estimates the average number of citations in good journal (inexed or listed in ISI, Scopus, ABDC, JQL, HEC journal list etc.) to recent articles published in that journal. To measure the importance of a journal within its field, journals with higher GSRI are deemed to be more important than those with lower ones. Inspiration for GSRI is derived from Eugene Garfield, the founder of the Institute for Scientific Information ISI Impact Factor. GSRI are calculated yearly starting from 2015 only for those journals that are published by Geist Science. Recently calculated GSRI for JMS is available on JMS website at http://geistscience.com/journals/jms . 